\title{
Avascular necrosis following closed reduction for treatment of developmental dysplasia of the hip: a systematic review
}

\author{
Catharine S. Bradley $^{1}$ - Daniel C. Perry ${ }^{2}$ John H. Wedge ${ }^{1}$ M. L. Murnaghan ${ }^{1}$. \\ Simon P. Kelley ${ }^{1}$
}

Received: 9 August 2016/Accepted: 21 October 2016/Published online: 3 November 2016

(c) The Author(s) 2016. This article is published with open access at Springerlink.com

\begin{abstract}
Background Avascular necrosis (AVN) is a significant and potentially devastating complication following the treatment of developmental dysplasia of the hip (DDH). The reported rate of AVN following closed reduction for DDH ranges from 4 to $60 \%$, and the resultant influence on hip development remains unclear.

Purpose A systematic review of the literature was undertaken to evaluate the frequency of AVN after more than 5 years of follow-up in children that underwent closed reduction at younger than 2-years of age for DDH.

Methods The search strategy was formulated with keyconcepts and keywords identified using the patient problem, intervention, comparison and outcome process. Searches were undertaken using Pubmed, Scopus and Web of
\end{abstract}

Electronic supplementary material The online version of this article (doi:10.1007/s11832-016-0776-y) contains supplementary material, which is available to authorized users.

Simon P. Kelley

simon.kelley@sickkids.ca

Catharine S. Bradley

catharine.bradley@ sickkids.ca

Daniel C. Perry

danperry@liv.ac.uk

John H. Wedge

john.wedge@ sickkids.ca

M. L. Murnaghan

lucas.murnaghan@sickkids.ca

1 Division of Orthopaedic Surgery, The Hospital for Sick Children, 555 University Avenue, Toronto, ON M5G 1X8, Canada

2 Institute of Translational Medicine, University of Liverpool, Eaton Road, Liverpool L12 2AP, UK
Science up to and including May, 2016 to identify potential studies.

Results A total of seven papers met the a priori inclusion and exclusion criteria of this review. The overall rate of significant AVN in 441 patients (538 hips) was $10 \%$ at a mean length of follow-up of 7.6 years (5-18.8) following closed reduction. This finding can be used to inform the feasibility of future intervention studies, and act as a baseline for which surgeons to compare their results to a 'standard'.

Keywords Developmental dysplasia of the hip . Congenital hip dislocation - Closed reduction - Avascular necrosis $\cdot$ Systematic review

\section{Introduction}

Closed reduction and spica casting is one of the most commonly performed procedures for the treatment of developmentally dislocated hips (DDH). Whilst the procedure may be considered 'minimally invasive' and commonplace, it is not without complications. The primary complication of closed reduction is avascular necrosis (AVN) of the femoral head that occurs due to diminished blood supply to the femoral epiphysis that can cause devastating clinical outcomes [1-7]. The aetiology of the AVN in closed reduction is thought to be a positional vascular occlusion in the spica cast, though the position-at-risk varies by child, and determination of this position cannot yet be routinely individualized. The 'optimal position in spica cast' therefore relies on a best-fit approach; using the safe-zone of Ramsey [8].

Reported frequency estimates of AVN following closed reduction vary significantly between 4 and 60\% [1-7]. 
There is therefore a significant discrepancy in the literature, with difficulty in interpreting the expected rate of AVN. Whilst this variation may be a consequence of natural variation due to the relatively small case numbers, it may similarly be due to systemic differences such as case selection, or surgical technique (i.e. cast position, duration of treatment, tendon release). A clear understanding of the expected AVN rate would empower surgeons to audit their own practice against a 'standard', would provide feasibility data for the measure of effect size in future intervention studies, and may help to elicit if there are particular aspects of the surgical intervention that may particularly heighten the risk of AVN.

The purpose of this study was to systematically evaluate the literature of children having underwent closed reduction under 2-years of age for DDH, to determine the frequency of AVN after more than 5-years of follow-up.

\section{Methods}

\section{Search strategy and criteria}

The search was conducted within Pubmed, Scopus and Web of Science up to and including May, 2016. The search strategy was formulated with key-concepts and keywords identified using the patient problem, intervention, comparison and outcome (PICO) process [9]. This identified essential search-terms, which were exploded ensuring the inclusion of relevant synonyms, alternative spellings and related terms [10]. Individual search terms were combined using Boolean technique to further refine the process. A medical librarian was instrumental in helping to design the search strategy.

Initial search keywords were broad and exploded terms, to ensure full use of MeSH (Medical Subject Headings) terms for maximum sensitivity. More specific terms and limitations were subsequently introduced and combined to refine the search [11] (Appendix 1).

For inclusion in the review, articles needed to be reports of clinical studies of DDH treated by closed reduction in human patients and a minimum series of ten hips. A minimum of ten hips was selected to minimize the effect of small sample bias in the overall analysis. The minimum follow-up period was 5 years, therefore this must either have been a feature of the study design, or the study must have published sufficient data such that individual cases with over 5 years of follow-up could be elicited. Shorter follow-up periods were not considered, as later-onset AVN, and more specifically type $2 \mathrm{AVN}$, that may not evident until several years after surgery, could not be excluded. Additionally, all studies had to report on AVN using a recognized and previously published classification of
AVN. Exclusion criteria were made if studies reported on closed reductions performed in children over 2 years of age (unless individual patient data was available to include such patients), and studies of children with teratologic hip dislocations (i.e. fixed hip dislocations at birth that are associated with congenital anomalies, other syndromes or neuromuscular disease) as closed reduction is typically not recommended in any of these subgroups. In addition, the search was limited to studies in English.

Titles and abstracts were independently screened against the inclusion criteria by two investigators with prior experience of conducting systematic reviews (XX and YY). If either investigator deemed that the title and abstract fulfilled the inclusion criteria, then the full paper was obtained. The same reviewers then screened the full papers against the inclusion and exclusion criteria and the reason that any paper failed to fulfill these criteria was noted. The senior author (ZZ) resolved any disagreement between the two reviewers. The reference lists of the included papers and of any identified review articles were also assessed for further relevant studies.

Data was extracted from those studies included independently by the two investigators using a Genaidy Critical Appraisal Instrument [12]. The detailed data from these forms were entered into an excel spreadsheet to allow for an assessment of heterogeneity and quality between studies.

\section{Outcome reporting}

Common AVN classifications systems have previously been combined in the following manner [13]: Type 1 AVN is identical in the Kalamchi and MacEwen, and Bucholz and Ogden classifications, but does not result in long term disease and has therefore not been considered clinically significant AVN. Type 2 AVN is also identical in both classifications and is thus considered together. Types 3 and 4 in each classification were recorded as clinically significant, and combined in a type 3 group. Salter classification-in instances where the location of physeal damage is reported, 'no physeal damage' is considered type 1, 'lateral physeal damage' is considered type 2 and 'central physeal damage' type 3 AVN. For the purposes of this study, only types 2-4 AVN are considered clinically significant and type $1 \mathrm{AVN}$ is not included in the analysis.

\section{Statistical analysis}

The overall frequency of and type of AVN (types 2-4) related to length of follow-up were evaluated across all included studies. Mean age at closed reduction in months, mean length of follow-up in years, percentages for gender and AVN classification system used were identified for each study. 


\section{Results}

The search identified 1492 possible titles and abstracts (Fig. 1). Initial review of these excluded 1382 articles and identified 28 review articles to further assess the included references. This resulted in the retrieval of 82 full papers for confirmation of eligibility. Searching references lists and conferring with experts did not add any further articles. Review of the full papers excluded an additional 72 articles leaving 7 articles (538 hips) for analysis. Four of these articles met inclusion criteria for all patients in their respective studies [14-17] and the remaining three provided sufficient individual patient data to be retrieved and included in the analysis [18-20].

Fig. 1 Literature search results

\section{Data extraction}

All seven studies were reports of case series and all but two $[14,19]$ were formed retrospectively. All studies reported outcomes of closed reduction for DDH including AVN. Exclusion criteria for the studies included teratologic hips and prior attempts at closed reduction. Most were conducted at a single institution, one at two medical centers [15] and one at four centers [16]. Summary details of each of the studies are provided in Table 1.

\section{Patient characteristics}

Of the 441 patients (538 hips), 86.5\% were female and $76.2 \%$ were unilateral in presentation. The mean age at

Titles and abstracts identified and screened $n=1492$

\begin{tabular}{|c|c} 
Excluded $\mathrm{n}=1382$ \\
Duplicates $n=8$ \\
Other language $n=3$ \\
Not relevant $n=184$ \\
Other outcome/intervention \\
$n=294$ \\
Other diagnoses $n=893$
\end{tabular}

\begin{tabular}{|c|c|}
$\begin{array}{c}\text { Review Articles identified } \\
\text { (full articles retrieved and } \\
\text { assessed for eligible } \\
\text { references) } n=28\end{array}$ \\
\hline
\end{tabular}

Full copies retrieved and assessed for eligibility $\mathrm{n}=82$

Additional studies identified from expert knowledge $\mathrm{n}=0$

Studies identified from searching in reference lists/review articles $\mathrm{n}=0$

Publications included in review $\mathrm{n}=7$ 
Table 1 Summary of included studies

\begin{tabular}{|c|c|c|c|c|c|c|c|}
\hline References & $\begin{array}{l}\text { Patients } \\
(n)\end{array}$ & $\begin{array}{l}\text { Affected } \\
\text { hips }(n)\end{array}$ & $\begin{array}{l}\text { Female } \\
(\%)\end{array}$ & $\begin{array}{l}\text { Mean age at } \\
\text { reduction (months) }\end{array}$ & $\begin{array}{l}\text { Mean follow-up } \\
\text { (years) }\end{array}$ & $\begin{array}{l}\text { AVN } \\
\text { classification }\end{array}$ & $\begin{array}{l}\text { Rate of significant } \\
\text { AVN (\%) }\end{array}$ \\
\hline Bicimoglu et al. [16] & 143 & 185 & 88.8 & $11.6(3-18)$ & $7.5(5-13)$ & $\mathrm{K} \& \mathrm{M}$ & $5.4(10 / 185)$ \\
\hline Carney et al. [18] & 32 & 35 & 77.1 & $8.2(1-21)$ & $8.75(5.3-13.6)$ & $\mathrm{B} \& \mathrm{O}$ & $37.1(13 / 35)$ \\
\hline Cooke et al. [15] & 42 & 48 & 92.9 & $10.2(2-20.6)$ & $11.1(5-18.8)$ & $\mathrm{K} \& \mathrm{M}$ & $2.1(1 / 48)$ \\
\hline Danielsson [19] & 65 & 67 & 89.4 & $10(2-20)$ & $11.7(6.2-18.2)$ & $\begin{array}{l}\text { Salter } \\
\text { K\&M }\end{array}$ & $6(4 / 67)$ \\
\hline Forlin et al. [20] & 28 & 33 & 89.3 & $13(3-21)$ & $7.3(5.3-12.6)$ & $\mathrm{K} \& \mathrm{M}$ & $6(2 / 33)$ \\
\hline Khoshhal et al. [14] & 85 & 124 & 69.4 & $7.3(3-14)$ & $\begin{array}{l}\text { "Minimum } 5 \text { years"-no } \\
\text { further details given. }\end{array}$ & $\mathrm{K} \& \mathrm{M}$ & $10.5(13 / 124)$ \\
\hline Pospischill et al. [17] & 46 & 46 & 83.3 & $4(1.2-10.4)$ & $6.3(5.2-7.2)$ & $\mathrm{B} \& \mathrm{O}$ & $19.6(9 / 46)$ \\
\hline
\end{tabular}

AVN classification; $K \& M$ Kalamchi and MacEwen, $B \& O$ Bucholz and Ogden

time of closed reduction was 9.6 months (range 1-21) with a mean follow-up of 7.6 years (range 5-18.8) after closed reduction.

\section{Interventions}

Previous treatment with a Pavlik harness was reported in three of the studies $[15,17,18]$ and pre-operative traction was used at varying rates in all but one study [16]. The closed reductions were performed either by a staff surgeon or under their supervision. Soft tissue releases were not routinely performed in all studies, but when completed, adductor longus was the only one noted. Reported time in spica ranged from 6 weeks [14] to 6 months [19]. Position of immobilization also ranged from a combined $100^{\circ}$ of flexion, $20^{\circ}$ of abduction [15] to combined flexion of $100^{\circ}-$ $110^{\circ}, 40^{\circ}-60^{\circ}$ of abduction and no internal rotation [17]. None reported using abduction greater than $60^{\circ}$.

\section{Overall rate of $\mathrm{AVN}$}

Significant AVN occurred in 52 of the 538 hips included (441 patients), which equates to an AVN rate of $10 \%$ for hips with a minimum of 5-years of follow-up [mean duration of follow-up of 7.7 years (range 5-18.8)].

\section{Time}

There was no apparent temporal relationship between AVN rate, and year of publication (Fig. 2). This suggests that there was no significant change in practice over the inclusion period, which significantly influenced AVN rates.

\section{Size of study}

There was a marked change in the AVN rate seen depending on the size of the study; which is largely a

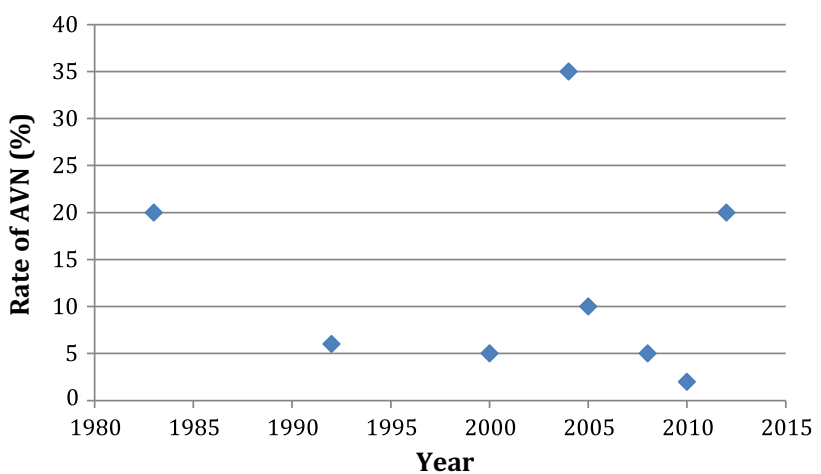

Fig. 2 Scatter plot illustrating the relationship between year of publication and the rate of types $2-4$ AVN

feature of common cause variation, with larger studies having more certainty, and therefore narrower confidence intervals. This is demonstrated using a funnel plot in Fig. 3. The funnel plot indicates that the target line for the predicted 'normal' rate of AVN (types 2-4) following closed reduction is $10 \%$. The control limits, drawn using three standard deviations, demonstrate that much of the variation within the published literature is explained by common cause variation, as the results lie within the control limits. One study fell significantly outside the control limits [18].

\section{Discussion}

This is the only review that has synthesized long-term AVN outcomes following closed reduction for Developmental Dysplasia of the Hip (DDH). This study has demonstrated that the mean rate of AVN within the literature is $10 \%$ after 5-years follow-up after closed reduction, amongst children treated before 2 years old. This result offers a 'target' against which clinicians may compare their results and offers a summary measure for use when powering intervention studies. 
The wide variations in the published results of AVN, is likely to be largely a consequence of common cause (natural) variation [21]. Common cause variation cannot be eliminated, and reflects the confidence or certainty within a sample. As the sample size is increased, the certainty increases, and the confidence interval narrows; hence a funnel is formed. Data points occurring outside the funnel are special cause variations, which indicate that an extrinsic cause is influencing the outcomes seen. Special cause variation may be due to differences in technique, surgeon factors or systematic differences in the way that a study is run. The result of one of the papers within this review fell significantly outside the control limits, indicating 'special cause variation' [18]. This may therefore indicate that there was a fundamental difference in some part of intervention offered to these participants within this study, or could be a consequence of a bias in the study design (i.e. cases with AVN may have been more readily identified and recruited owing to more frequent follow-up visits). Such special cause variations require further investigation in order to identify and act upon the special cause.

With increasing transparency within surgery, it is important that surgeons are able to audit their results against a gold standard by which they can benchmark. Likewise, it is also important that they understand the concept of natural variation, such that they are able to consider their results within the context of the number of procedures that they undertake; thereby ensuring that their benchmarking is appropriate. Internationally, arthroplasty surgeons are perhaps most used to this type of scrutiny, as the volumes by which they undertake procedures sufficiently narrows confidence intervals in order to readily identify outliers and act accordingly. Whilst this may be somewhat more difficult within paediatric orthopaedics, efforts to increase transparency and audit results (by individual or centre) should be encouraged.

There may be specific aspects of the intervention that may have a bearing on outcome that cannot be well ascertained given the nature of this review and the included studies. Other previous studies, not included within this review, have made observations, particularly regarding the importance of the presence of the ossific nucleus prior to closed reduction [22, 23], though it remains controversial [24]. In addition, there may be adaptions within the surgical technique, such as routine adductor tenotomy and duration and position of immobilization in spica casting that may minimize the complication of AVN. This suggestion warrants that well designed prospective analysis of potential predictive factors. In association with the International Hip Dysplasia Institute (IHDI), there is an ongoing

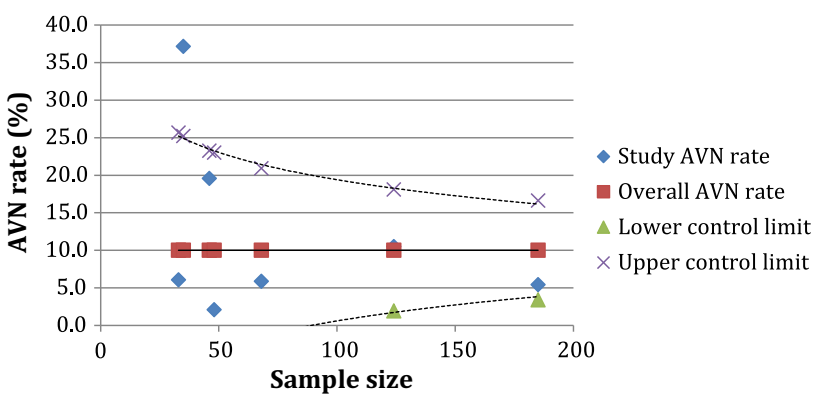

Fig. 3 Funnel plot to illustrate the rate of types 2-4 AVN, according to study sample size

multicentre nationwide trial within the UK that is seeking to address the timing of the intervention (i.e. immediate vs. delayed after the appearance of the ossific nucleus). The results of this, and other similar trials, may have profound effects for the way that we manage DDH [25].

A systematic review such as this, attempts to harmonize the results of a number of studies, by strictly defining inclusion/exclusion and intervention variables. Whilst this is useful to gain a summary measure, flaws within individual studies are difficult to overcome. These flaws are particularly apparent for retrospective case series, whereby the population was never clearly defined, case ascertainment was unclear, potential confounders were not recorded (i.e. position in spica) and the methodological clarity was limited. The review was also limited by the analysis of only literature written in English, and the paucity of studies that have reported long-term follow-up. Nevertheless, the study of rare outcomes is challenging in a prospective manner [26], owing to the costs and infrastructure required to identify and follow-up cases in a systematic manner, therefore a pragmatic approach must be considered.

This review has enabled individual surgeons/centres to benchmark themselves against the most robust studies of outcome within the literature and has set a summary measure against which future studies may compare outcomes.

Acknowledgements The authors would like to thank Ken Linkman, Medical Librarian, Henry Cohen Library, University of Liverpool for his assistance in conducting the literature search component of this study.

\section{Compliance with ethical standards}

Conflict of interest Catharine S. Bradley, Daniel C. Perry and M.L. Murnaghan declare that they have no conflict of interest. John H. Wedge and Simon P. Kelley are members of the Medical Advisory Board of the International Hip Dysplasia Institute.

Ethical approval This article does not contain any studies with human participants or animals performed by any of the authors. 
Open Access This article is distributed under the terms of the Creative Commons Attribution 4.0 International License (http://crea tivecommons.org/licenses/by/4.0/), which permits unrestricted use, distribution, and reproduction in any medium, provided you give appropriate credit to the original author(s) and the source, provide a link to the Creative Commons license, and indicate if changes were made.

\section{References}

1. DeRosa GP, Feller N (1987) Treatment of congenital dislocation of the hip. Management before walking age. Clin Orthop Relat Res 225:77-85

2. Joseph K, MacEwen GD, Boos ML et al (1982) Home traction in the management of congenital dislocation of the hip. Clin Orthop Relat Res 165:83-90

3. Kahle WK, Anderson MB, Alpert J et al (1990) The value of preliminary traction in the treatment of congenital dislocation of the hip. J Bone Jt Surg Am 72(7):1043-1047

4. Quinn RH, Renshaw TS, DeLuca PA et al (1994) Preliminary traction in the treatment of developmental dislocation of the hip. J Pediatr Orthop 14(5):636-642

5. Race C, Herring JA (1983) Congenital dislocation of the hip: an evaluation of closed reduction. J Pediatr Orthop 3(2):166-172

6. Zionts LE, MacEwen GD (1986) Treatment of congenital dislocation of the hip in children between the ages of one and three years. J Bone Jt Surg Am 68(6):829-846

7. Malvitz TA, Weinstein SL (1994) Closed reduction for congenital dysplasia of the hip. Functional and radiographic results after an average of thirty years. J Bone Jt Surg Am 76(12):1777-1792

8. Ramsey PL, Lasser S, MacEwen GD et al (1976) Congenital dislocation of the hip. Use of the Pavlik harness in the child during the first six months of life. J Bone Jt Surg Am 58(7):1000-1004

9. Sackett DL, Rosenberg WM, Gray JA et al (1996) Evidence based medicine: what it is and what it isn't. BMJ 312(7023):71-72

10. Oxman AD, Sackett DL, Guyatt GH et al (1993) Users' guides to the medical literature. I. How to get started. The Evidence-Based Medicine Working Group. JAMA 270(17):2093-2095

11. Doig GS, Simpson F (2003) Efficient literature searching: a core skill for the practice of evidence-based medicine. Intensive Care Med 29(12):2119-2127

12. Genaidy AM, Lemasters GK, Lockey J et al (2007) An epidemiological appraisal instrument-a tool for evaluation of epidemiological studies. Ergonomics 50(6):920-960

13. Gardner ROE, Bradley CS, Howard A et al (2014) The incidence of avascular necrosis and the radiographic outcome following medial open reduction in children with developmental dysplasia of the hip: a systematic review. Bone Jt J 96(B(2)):279-286

14. Khoshhal KI, Kremli MK, Zamzam MM et al (2005) The role of arthrography-guided closed reduction in minimizing the incidence of avascular necrosis in developmental dysplasia of the hip. J Pediatr Orthop B 14(4):256-261

15. Cooke SJ, Rees R, Edwards DL et al (2010) Ossification of the femoral head at closed reduction for developmental dysplasia of the hip and its influence on the long-term outcome. J Pediatr Orthop B 19(1):22-26

16. Biçimoğlu A, Ağuş H, Omeroğlu H et al (2008) Posteromedial limited surgery in developmental dysplasia of the hip. Clin Orthop Relat Res 466(4):847-855

17. Pospischill R, Weninger J, Ganger R et al (2012) Does open reduction of the developmental dislocated hip increase the risk of osteonecrosis? Clin Orthop Relat Res 470(1):250-260

18. Carney BT, Clark D, Minter CL et al (2004) Is the absence of the ossific nucleus prognostic for avascular necrosis after closed reduction of developmental dysplasia of the hip? J Surg Orthop Adv 13(1):24-29

19. Danielsson L (2000) Late-diagnosed DDH: a prospective 11-year follow-up of 71 consecutive patients (75 hips). Acta Orthop Scand 71(3):232-242

20. Forlin E, Choi IH, Guille JT et al (1992) Prognostic factors in congenital dislocation of the hip treated with closed reduction. The importance of arthrographic evaluation. J Bone Jt Surg Am 74(8):1140-1152

21. Perry DC, Parsons N, Costa ML et al (2013) Surgeon level data: understanding the plot. Bone Jt J 95(B(9)):1156-1157

22. Roposch A, Stöhr KK, Dobson M et al (2009) The effect of the femoral head ossific nucleus in the treatment of developmental dysplasia of the hip. A meta-analysis. J Bone Jt Surg Am 91(4):911-918

23. Clarke NMP, Jowett AJL, Parker L et al (2005) The surgical treatment of established congenital dislocation of the hip: results of surgery after planned delayed intervention following the appearance of the capital femoral ossific nucleus. J Pediatr Orthop 25(4):434-439

24. Roposch A, Odeh O, Doria AS et al (2011) The presence of an ossific nucleus does not protect against osteonecrosis after treatment of developmental dysplasia of the hip. Clin Orthop Relat Res 469(10):2838-2845

25. Hip Op Trial (2015) Timing of Surgical Intervention for Developmental Dysplasia of the Hip. Clarke NMP. 2014. Available from: http://www.nets.nihr.ac.uk/_data/assets/pdf_file/0003/ 123348/PRO-11-146-01.pdf. Accessed April 2015

26. Dietz FR (2010) Reflections on the failure of the POSNA-sponsored multicenter trial for treatment of unstable slipped capital femoral epiphysis. J Pediatr Orthop 30(4):386-389 